\title{
A Control Strategy of a Single-phase H6-type Grid-connected Inverter with Low Harmonic Current
}

\author{
Lin Chunxu ${ }^{1}$, Zhou Chunhua ${ }^{1}, \mathrm{Li} \mathrm{Wei}^{1}$, Chen Rui ${ }^{1}$ \\ ${ }^{1}$ Department of Electrical Engineering and information, Southwest Petroleum University, Chengdu Sichuan, China
}

\begin{abstract}
In order to reduce the total harmonic distortion (THD) of the grid-connected current caused by the high-frequency switching of the inverter, this paper combines the high efficiency single-phase H6-type inverter with LCL filter. The double closed-loop control method that consists of grid-connected current outer loop and capacitor current inner loop is put forward, by which a resonance peak of a low damping LCL filter is eliminated. In the grid-connected current outer loop, quasi proportion resonant (QPR) controller is adopted to overcome the steady-state error and weak anti-jamming capability in traditional PI controller. Finally, a simulation model is built in SIMULINK to verify the research. The simulation results show that, based on the single-phase H6-type inverter and LCL filter, the double closed-loop QPR control strategy can achieve the static error free tracking control of grid-connected current, which makes the system more stable and reduces the THD of grid-connected current effectively.
\end{abstract}

\section{Introduction}

With the development of new energy industry and smart micro-grid, inverters have drawn more and more attention as the core of grid-connected system. Ensuring the output quality of inverters and reducing the total harmonic distortion (THD) have also attracted wide attention from domestic and foreign scholars (Wang Y. et al, 2014).

Compared with the traditional H4-type inverter, the H6-type inverter adds two full-controlled power switches and two high-performance diodes. In the free-wheeling stage, after the poor performance body diodes are replaced by these high-performance diodes, the system has higher efficiency (Ji B. et al, 2012). At the same time, the power grid and the DC battery board are isolated, which doesn't need the isolating transformer. And when using appropriate control strategy and modulation method, the leakage current can be restrained effectively. Therefore the H6-type inverter is used in various high-power occasions widely.

Recently the H6-type inverter mostly adopts proportional (P) controller, proportional integral (PI) controller (Dai L. \& Long Y-p., 2012) or proportional resonance (PR) controller (Wang Y. et al, 2016). The P controller is too simple to meet the fast response requirements of the system. In terms of signal tracking, the PI controller has some defects that are steady-state error and weak anti-jamming capability. The PR controller not only solves the static error that can't be eliminated by the traditional PI controller, but also restrains the disturbance caused by the voltage of the power grid. However its disadvantage is that the frequency cannot be adaptive, which will cause greater harmonic interference with the deviation of grid frequency.

In order to reduce the influence of high-frequency switching harmonics current on grid-connected current, the traditional grid- connected inverter is usually connected to the power grid with an $\mathrm{L}$ filter. The inductance value of the $\mathrm{L}$ filter is large, which leads to the increase of the inductance pressure drop, the wastage and the cost. What's worse, the response speed of the current inner loop is affected to a certain extent, and the traditional L filter hasn't met the requirement in particular at higher power places. On the other hand, LCL filter shows high-impedance characteristic on high-frequency components (Wang D. et al, 2012), and choosing a smaller inductance can greatly attenuate high-frequency harmonics current, which has also better filter performance than single inductance. However, as an undamped three-order system, the resonance phenomenon of LCL filter can lead to the instability of the system. Therefore some methods are put forward these years (Zhuang C. et al, 2015; Sun W. et al, 2008). However, those methods either need compensation control or cause new wastage, and these inverter topologies are ordinary H4-type inverters. As for the more efficient single-phase H6-type inverter, there are a few studies on using LCL filter for grid-connected inverter.

In order to solve the above problems, this paper first analyzes the topology of a single-phase H6-type grid-connected inverter with LCL filter (H6LCL for short). Then, the stability of the system is explored by using the QPR controller and the double current closed-loop control which consists of grid-connected current outer loop and capacitor current inner loop. Finally, the simulation results of SIMULINK platform

\footnotetext{
*Corresponding author:Chunxu28@foxmail.com, \{zhizihua328, 1084178944,1571495993\}@qq.com
} 
verify the rationality of the proposed control strategy.

\section{Topology Of The H6LCL System}

The topology of the H6LCL system is shown as Figure 1, where $E$ is a DC input voltage, $S_{1} \sim S_{6}$ are IGBTs, $D_{1}$ and $D_{2}$ are the free-wheeling diodes. LCL filter consists of $L_{1}$, $L_{2}$ and $\mathrm{C}$ (ignoring the parasitic resistances of the inductance and the capacitance). $i_{1}$ is the inductance current on the side of the inverter, $i_{\mathrm{c}}$ is the current through the filter capacitor $C, i_{2}$ is the inductor current on the side of the power grid, $u_{\mathrm{c}}$ is the voltage of the filter capacitor $\mathrm{C}$ and $u_{\mathrm{g}}$ is the grid voltage.

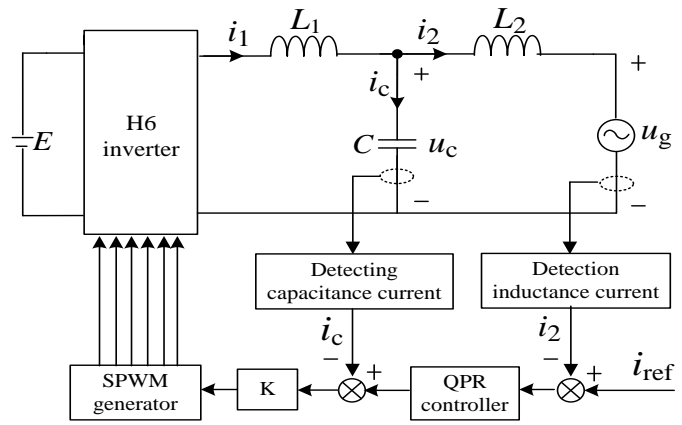

Figure 1. The topology of H6LCL

The control strategy is explained as follows. Firstly, comparing the reference current $i_{\text {ref }}$ with the grid-connected current $i_{2}$, the obtained error signal is sent into the QPR controller, then the signal is compared with the capacitor current in the inner loop. Through coefficient adjustment, the inner loop error signal is sent into the SPWM signal generator, and the SPWM wave works on the power switches of the H6-type grid-connected inverter to realize the grid-connected control.

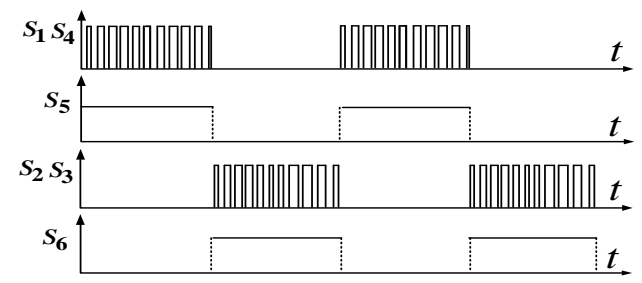

Figure 2. The modulation wave of H6LCL

The unipolar modulation mode is adopted in the H6LCL system, whose waves are shown in Figure 2. $S_{1} \sim S_{4}$ work in high-frequency switching state, $S_{5}$ and $S_{6}$ work in power-frequency switching state. The PWM driver signals of $S_{1}$ and $S_{4}$ are identical, the PWM driver signal of $S_{2}$ is equal to that of $S_{3}$, and the two driving signals complement each other on a cycle. From Figure 1 and Figure 2, $S_{5}$ and $D_{2}, S_{6}$ and $D_{1}$ are two free-wheeling channels of the H6LCL inverter, which separate the DC battery board from the power grid. After the body diodes with poor performance are replaced by fast recovery diodes, the reverse recovery wastage of diodes can be reduced effectively, therefore the overall efficiency of the system is greatly improved.

\section{Double closed-loop and QPR control strategy}

\subsection{QPR control}

In the traditional grid-connected inverter, the defects of steady-state error and poor anti- interference ability make the PI controller limited. The PR controller adds a lossless resonant part in the process of controlling the inverter output. The gain is infinite and the phase is almost equal to 0 at the resonance frequency, so the steady-state error of the system can be eliminated. In the H6LCL system, the resonant frequency is $50 \mathrm{~Hz}$ (equal to the grid voltage frequency), and the transfer function of PR controller is shown:

$$
G_{\mathrm{PR}}(s)=\mathrm{K}_{\mathrm{P}}+\frac{2 \mathrm{~K}_{\mathrm{R}} s}{s^{2}+\omega_{0}^{2}}
$$

The bode plot of PR controller is shown in Figure 3, which indicates that using PR controller can greatly obtain great gain at the frequency of fundamental wave. Especially at the frequency $50 \mathrm{~Hz}(314 \mathrm{rad} / \mathrm{s})$, the gain can be considered as infinity.

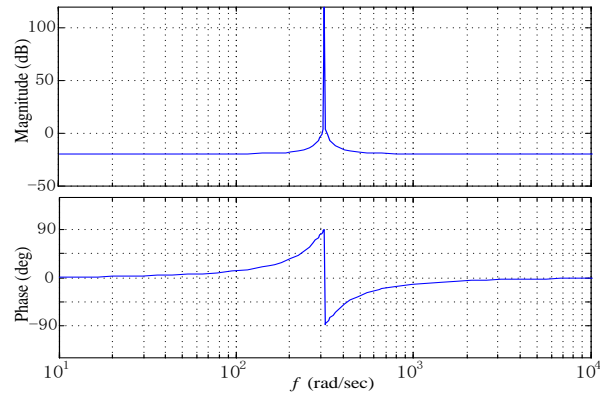

Figure 3. The bode plot of PR controller

If the power grid frequency always maintains at this ideal point, harmonics can be suppressed effectively by the PR controller and a better grid-connected current can be obtained. Normally the frequency of the power grid, however, will deviate about $0.1 \mathrm{~Hz} \sim 0.2 \mathrm{~Hz}$, which will greatly reduce the ability of the PR controller to suppress the harmonics. In order to solve the problem and reduce the sensitivity of the system due to the frequency deviation of the power grid, the QPR control strategy is adopted. The transfer function can be expressed as

$$
G_{\mathrm{QPR}}(s)=\mathrm{K}_{\mathrm{P}}+\frac{2 \mathrm{~K}_{\mathrm{R}} \omega_{\mathrm{c}} s}{s^{2}+2 \omega_{\mathrm{c}} s+\omega_{0}^{2}}
$$

Where the proportional parameter $\mathrm{K}_{\mathrm{p}}=0.5$, the resonance parameter $K_{R}=10$, the angular frequency of the resonant fundamental wave $\omega_{0}=314 \mathrm{rad} / \mathrm{s}$, and the bandwidth $\omega_{\mathrm{c}}=3.14 \mathrm{rad} / \mathrm{s}$. The bode plot, as shown in Figure 6, is drawn by Eq.(2). Compared with Figure 3, the bandwidth of the system has increased near the resonant frequency when QPR controller is adopted. It can effectively solve the problem that PR controller can't restrain harmonic interference while grid frequency deviating. Therefore, the ability of the system resisting grid voltage disturbance is also enhanced. 


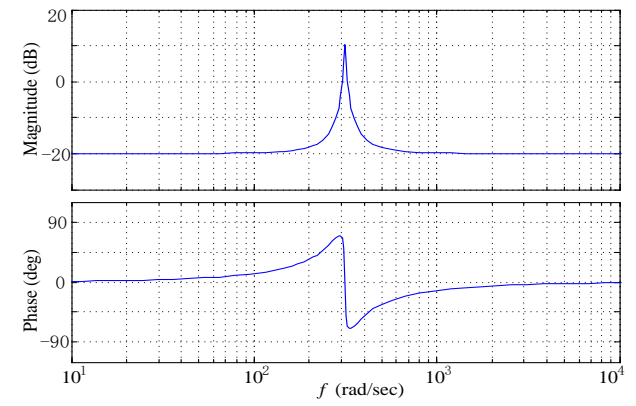

Figure 4. The bode plot of QPR controller.

\subsection{Double current closed-loop control}

The dynamic response of LCL filter is superior and has a better harmonic suppression performance than $\mathrm{L}$ filter. But, as a third-order system, the lower system damping of LCL filter results in the resonance peak at the natural frequency. In order to improve the stability of control system, the system oscillation must be suppressed. However, the resonance of LCL filter is caused by the lower system damping. To this end, a double current closed-loop control strategy that consists of grid-connected current outer loop and capacitor current inner loop, is introduced. And the closed-loop control block diagram, as shown in Figure 5(a), can be obtained by Figure 1. Then Figure 5(a) is simplified into Figure 5(b) to further obtain the transfer function of the closed-loop control system.

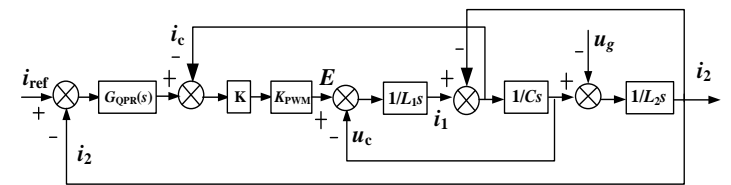

(a)

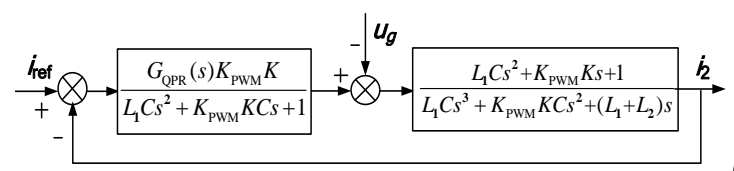

Figure 5. The double closed-loop control block diagram of capacitor current and grid-connected current

Finally the transfer function of the double current closed-loop control in Figure 5(b) is shown as Eq. (3):

$$
\phi(s)=\frac{\boldsymbol{i}_{2}}{\boldsymbol{i}_{\mathrm{ref}}}(s)=\frac{G_{\mathrm{QPR}}(s) \mathrm{K}_{\mathrm{PWM}} \mathrm{K}}{L_{1} L_{2} C s^{3}+L_{2} C \mathrm{~K}_{\mathrm{PWM}} \mathrm{K}^{2}+\left(L_{1}+L_{2}\right) s+G_{\mathrm{PPR}}(s) \mathrm{K}_{\mathrm{PWM}} \mathrm{K}}
$$

Where $\mathrm{K}_{\mathrm{PWM}}$ is the equivalent section of H6-bridge, and $\mathrm{K}$ is the proportional coefficient of inner loop channel. According to the research (Li M. et al, 2010), $\mathrm{K}_{\mathrm{PWM}}=40, \mathrm{~K}=0.86$. As shown in Figure 6, the bode plot of the double closed-loop control can be drawn by Eq. (2) and Eq. (3). Compared with Figure 3, the double closed-loop control strategy of capacitor current and grid-connected current can effectively inhibit the resonance peak of LCL filter at the natural frequency, and have great harmonic suppression performance in high-frequency bandwidth.

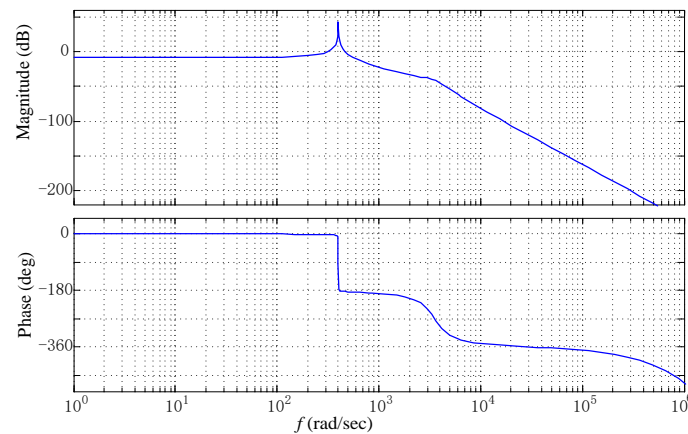

Figure 6. The bode plot of the double current closed-loop control.

\section{Simulation verification}

In order to verify the correctness of the above analysis and control strategy, the simulation model is set up on the Matlab/SIMULINK. The circuit parameters of the H6LCL system are described as Table.1.

Table1. Circuit parameters of the H6LCL system

\begin{tabular}{|c|c|}
\hline Parameter & Value \\
\hline DC voltage $E$ & $400 \mathrm{~V}$ \\
\hline RMS of grid voltage $U_{\mathrm{g}}$ & $220 \mathrm{~V}$ \\
\hline reference current $i_{\text {ref }}$ & $25 \mathrm{~A}$ \\
\hline filter inductor $L_{1}$ & $2 \mathrm{mH}$ \\
\hline filter inductor $L_{2}$ & $0.4 \mathrm{mH}$ \\
\hline filter capacitor $C$ & $14 \mu \mathrm{F}$ \\
\hline grid voltage frequency $f$ & $50 \mathrm{~Hz}$ \\
\hline switching frequency $f_{\mathrm{k}}$ & $16 \mathrm{KHz}$ \\
\hline sampling frequency $f_{\mathrm{s}}$ & $50 \mathrm{KHz}$ \\
\hline
\end{tabular}

The simulation results are shown as follows: Figure 7(a) is the waves of grid-connected current (amplified 10 times) and grid-connected voltage, Figure $7(\mathrm{~b})$ is the waves of grid-connected current and reference current wave, and Figure 8 is the THD of grid-connected current.

As can be seen from Figure 7(a) and Figure 7(b), based on the single-phase H6-type inverter and LCL filter, the double closed-loop QPR control strategy can achieve the static error free tracking control on grid-connected current, which makes the system more stable. The phase of grid-connected voltage is almost the same as that of grid-connected current, therefore the power factor of the system is close to 1 .

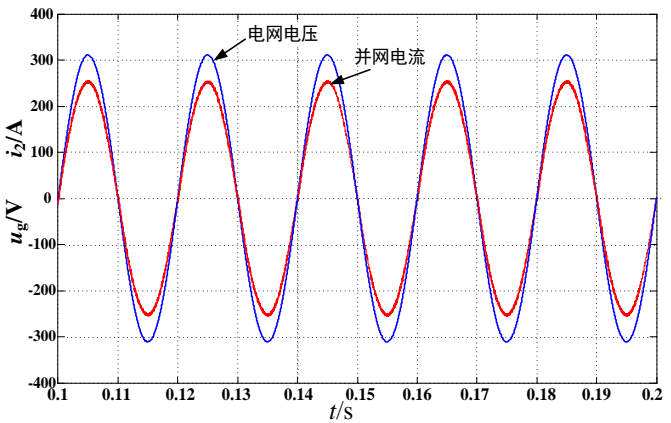

(a) 


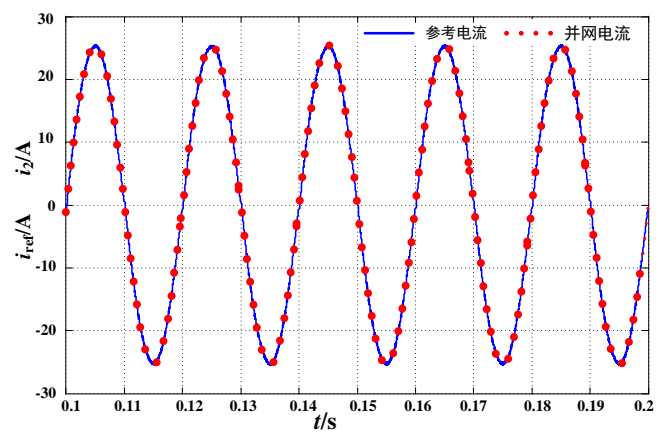

(b)

Figure 7. The simulation results: (a) The waves of grid-connected current (amplified 10 times) and grid-connected voltage; (b) The waves of grid-connected current and reference current wave

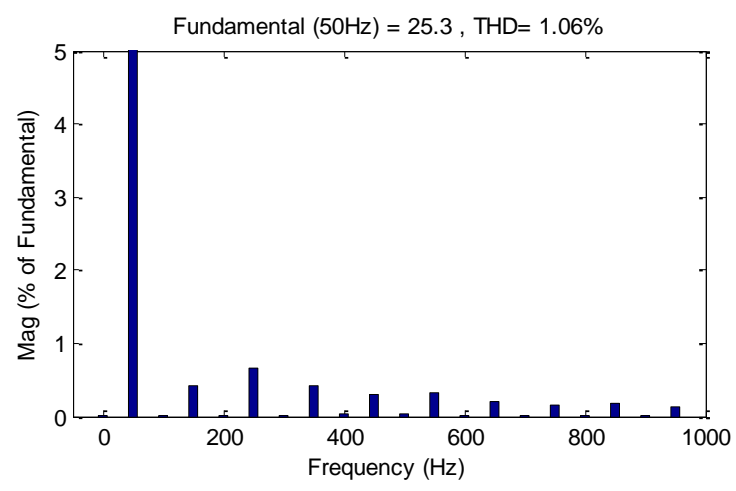

Figure 8. The THD of grid-connected current

The FFT analysis result of the five cycles' wave of the grid-connected current is shown in Figure 10, which states clearly the THD of the grid-connected current is only $1.06 \%$. Above simulation results testify that the H6LCL system has a superior ability to filter and suppress the high-frequency harmonic effectively.

\section{Conclusion}

The high efficiency single-phase H6-type inverter with LCL filter can obtain better electrical energy than the traditional inverter. Aimed at the problem that the oscillation of the third-order LCL filter causes the instability of the system, an active damping control scheme is adopted in this paper. The double current closed-loop control method is put forward, and the QPR control strategy is introduced in the outer loop of the grid-connected current. Through the design and simulation of the $4 \mathrm{KW}$ inverter, the control strategy can suppress the resonance of the natural frequency effectively, realize zero steady-state error and improve the stability of the system. The simulation results also show that the strategy can be used to filter the high-frequency harmonics when using a small filter inductance, which reduces the system cost and get the THD of the grid-connected current only $1.06 \%$, so the grid quality has improved further.

\section{Acknowledgements}

The authors gratefully acknowledge the financial supports by the National innovation and Entrepreneurship Program of China under grant numbers (201710615007).

\section{References}

1. Dai L., Long Y-p., 2012. Study of bifurcation and chaos for photovoltaic inverter with PI controller, Power System Protection and Control, 24, P89-94.

2. Li M., et al., 2010. Research of three-loop control method of photovoltaic grid-connected inverter. Power System Protection and Control, 38(19), p.46-50.

3. Ji B., et al., 2012. A High Efficiency Non-isolated Single-phase Photovoltaic Grid-connected Inverter With H6-type. Chinese Journal of Electrical Engineering, 32(18), p.9-15.

4. Sun W., et al., 2008. An Overview of Current Control Strategy for Three-Phase Voltage-Source Rectifier With LCL-Filter. Transactions of China Electrotechnical Society, 23(1), p.90-96.

5. Wang, Y., et al., 2016. Control strategy for grid-connected inverter to suppress harmonics based on PR regulator. Power Supply Technology, 40(1), p.184-188.

6. Wang Y., et al., 2014. New Harmonic Damping Strategy for Single Grid-connected Inverter With LCL Filter. Chinese Journal of Electrical Engineering, 33, p.5803-5810.

7. Wang D., et al., 2012. The Simulation of Double-Loop Grid Current Control Technique for Grid-Connected Inverter Using Single LCL Filter. Communication Power Technology, 29(02), p.5-7.

8. Zhuang C., et al., 2015. Analysis and Improvement of the Control Strategy of LCL Grid-Connected Inverter Based on Split-Capacitor. Transactions of China Electrotechnical Society, 16, p.85-93. 